\title{
APP DISEÑADA PARA EL ENTRENAMIENTO DE LA MATEMÁTICA TEMPRANA. LA ESTIMACIÓN EN LA RECTA NUMÉRICA
}

\author{
Carlos Mera Cantillo \\ Universidad de Cádiz (España) \\ carlos.mera@uca.es \\ Gonzalo Ruiz Cagigas \\ Universidad de Cádiz (España) \\ José Ignacio Navarro Guzmán \\ Universidad de Cádiz (España) \\ Belén Román Alegre \\ Universidad de Cádiz (España) \\ Estíbaliz Aragón Mendizábal \\ Universidad de Cádiz (España)
}

\section{RESUMEN}

Actualmente, venimos escuchando en diversos foros educativos y científicos la necesidad de trabajar en la mejora del aprendizaje matemático del alumnado. Las matemáticas son consideradas por los estudiantes unas de las materias más complejas para su comprensión. También son de las materias que presentan unos resultados menos alentadores en las calificaciones de nuestros estudiantes más jóvenes. Las investigaciones que se han centrado en el desarrollo de la competencia matemática, han destacado la necesidad de realizar una intervención temprana. Asimismo, es necesario facilitar el aprendizaje utilizando herramientas que puedan resultar atractivas a los alumnos, como puede ser el uso de las nuevas tecnologías para fines educativos. Por otro lado, la utilización de dispositivos electrónicos de uso muy extendido como tablets o smartphones pueden generar un acercamiento de la educación al entorno familiar de nuestros jóvenes, de tal manera que el niño puede acceder al aprendizaje mientras juega en casa. En este trabajo, se presenta una de las aplicaciones ( $\underline{\text { APPS }}$ ) diseñadas para tablet y smartphone adaptada a los diferentes sistemas operativos actuales. Están diseñadas y desarrolladas para incidir sobre la mejora del sentido numérico del niño, y destinadas al alumnado en sus primeras etapas educativas. La APP que se presenta tiene como objetivo el entrenamiento de la "estimación en la recta numérica", siendo esta habilidad uno de los principales precursores específicos del aprendizaje en matemáticas. Esta APP se fundamenta en los modelos cognitivos de aprendizaje numérico, y a su vez aporta los beneficios derivados del uso de 
uno de los instrumentos más utilizados como reforzadores en la actualidad en el entorno familiar, como son las tablets y los smartphones. El objetivo principal del trabajo es contribuir en el desarrollo de las habilidades matemáticas de los niños/as de 4 a 7 años para afrontar con éxito los requerimientos de la escuela y resolver situaciones en sus vidas cotidianas.

Palabras clave: sentido numérico, APPs, estimación

\section{ABSTRACT}

An APP designed for early mathematics training. The number line estimation. Currently, we have been hearing from various educational and scientific forums the need to improve students' mathematical learning. Mathematics is considered one of the most complex topics for comprehension. Math also is the subject presenting lowest grades of our students. Research focused on of mathematical competence development, has highlighted the need to implement early intervention to improve further progress. Likewise, it is necessary to facilitate learning using tools that may be attractive to students, such as the use of new technologies for educational purposes. The use of high tech devices such as tablets or smartphones can approach education in the family environment of our young people. In such a way that children can learn while they are playing at home. In this work, we present one of the applications (APP) designed for tablet and smartphone, adapted to the different platforms. App is designed and developed for improvement children's numerical sense. Apps is designed for Early Childhood Education. This APP is is focused on training number line estimation. This skill is one of the main specific mathematics learning precursor. APP is based on cognitive models of numerical learning, and provides the benefits derived from the use of one of the tools used as reinforces by families (tablets and smartphones). This work would contribute to the development of mathematical skills of children aged from 4 to 7 so that they can successfully face the school requirements for maths, solve daily lives features.

Keywords: number sense, APPs, estimation

\section{ANTECEDENTES}

La asignatura de matemáticas, sigue generando cierto grado de dificultad entre los estudiantes de nuestro país. De hecho, según datos del último informe PISA (2015) en cuanto al desempeño de nuestros alumnos en matemáticas, obtenemos unas puntuaciones por debajo del nivel de países con nuestro mismo nivel socioeconómico. De esta forma, los resultados de estudiantes españoles presentan diferencias considerables con respecto al promedio de los de otros países de la OCDE. En el año 2006, uno de los mayores grupos de recursos humanos y empleo de nuestro país, ha presentado un nuevo documento que viene a informarnos que el futuro del empleo, y el bienestar de nuestros hijos, pasa por la tecnología, la ciencia, las ingenierías y las matemáticas, si bien, los jóvenes españoles se decantan en mayor medida por las carreras de letras con menos salidas profesionales dando así la espalda a los números (Randstad, 2016). Además de esto, se estima que una proporción significativa del alumnado presenta dificultades en la aritmética, siendo identificados como alumnos con dificultades de aprendizaje matemático, o discalculia (Desoete, Roeyers, \& De Clercq, 2004). Para conocer sus perfiles cognitivos y las posibilidades de rehabilitación, se han propuesto varias tipologías de niños con discalculia (Bartelet, Vaessen, Blomert, \& Ansari, 2014; Geary, 2004; Von Aster, 2000). La investigación ha establecido que el conocimiento matemático temprano de los niños predice fuertemente su éxito posterior en matemáticas (Clements \& Sarama, 2009). Es importante tener en cuenta que el desarrollo matemático del niño en la etapa preescolar predice su rendimiento posterior incluso en la enseñanza universitaria. También predice el logro posterior en lectura incluso mejor que las habilidades de lectura temprana (Solsona, Navarro, \& Aguilar, 2009). De hecho, la investigación muestra que un buen desarrollo matemático aumenta las habilidades de len- 
guaje (Denton, West, \& Walston, 2003; Nguyen et al., 2016). Las destrezas matemáticas y cognitivas son un elemento clave para el desarrollo de los países y el crecimiento económico (Joensen \& Nielsen, 2010; Muñoz, Okan, \& Garcia-Retamero, 2015; OECD, 2016). Debido a la relación que existe entre los problemas de aprendizaje en matemática temprana y el posterior desarrollo académico y profesional del niño, es especialmente importante intentar comprender mejor las señales tempranas de las dificultades de aprendizaje matemático, tanto para la prevención como para la intervención, ya que los problemas asociados a estas dificultades son persistentes en el tiempo (Shalev, Manor, \& Gross-Tsur, 2005). Dada, la importancia de las matemáticas para el éxito académico en todas las materias, todos los niños necesitan adquirir y desarrollar un conocimiento sólido de las matemáticas en sus primeros años de escolarización.

Los estudios en psicología cognitiva y del desarrollo siguen centrados en el estudio de los factores cognitivos asociados al aprendizaje de la matemática temprana desde dos líneas de investigación. Una primera línea se centra principalmente en los denominados precursores de dominio general, tales como la memoria de trabajo (Baddeley, 2000; Baddeley \& Hitch, 1974; Vujic, 2017), la memoria a corto plazo, la velocidad de procesamiento y la inteligencia. Estos procesos cognitivos se consideran precursores de la competencia matemática, además de predecir otras destrezas relacionadas con diversas materias educativas (Aragón, Navarro, Aguilar, \& Cerda, 2014; Cirino, 2011; Gullick, Sprute, \& Temple, 2011; Navarro, Aguilar, Marchena, Ruiz, \& Ramiro, 2011; Nayfeld, Fuccillo, \& Greenfield, 2013; Purpura \& Ganley, 2014). Por otro lado, una segunda línea se basa en el estudio de los denominados precursores de dominio específico de la habilidad matemática, generalmente agrupados bajo el constructo "sentido numérico" (Cowan \& Powell, 2014; Geary, Hamson, \& Hoard, 2000; Hannula, Lepola, \& Lehtinen, 2010; Krajewski \& Schneider, 2009; E. H. Kroesbergen, Van Luit, Van Lieshout, Van Loosbroek, \& Van De Rijt, 2009). Un déficit en el sentido numérico sería una de las causas principales de la aparición de Dificultades de Aprendizaje Matemático (DAM) (van Viersen, Slot, Kroesbergen, van't Noordende, \& Leseman, 2013). Se han considerado varios componentes del sentido numérico como factor clave en el desarrollo de la habilidad matemática o la falta de la misma, tal como el sentido simbólico y/o no simbólico del número (De Smedt \& Gilmore, 2011; Mussolin, Mejias, \& Noël, 2010; Sasanguie, Van den Bussche, \& Reynvoet, 2012), o la relación entre estos dos sistemas cuantitativos (Kolkman, Kroesbergen, \& Leseman, 2013; Rousselle \& Noël, 2007).

Uno de los predictores cognitivos de dominio especifico con mayor relevancia en el futuro desarrollo de la aritmética en edades tempranas es la capacidad de estimar una cantidad simbólica y situarla en su orden numérico adecuado, la estimación en la recta numérica (Aragón et al., 2014; Siegler \& Booth, 2004; Zhu, Cai, \& Leung, 2017). Esta acción se puede realizar de dos maneras diferentes, presentando una línea y pidiendo que coloque un número concreto en el lugar donde corresponde (número-posición) o proporcionando una línea con un lugar señalado en la misma y preguntando a que número corresponde (posición-número) (Laski \& Siegler, 2007; Núñez-Peña, Colomé, \& Aguilar-Lleyda, 2019; Siegler \& Booth, 2004)

El uso del ordenador como herramienta educativa es previo a la aparición de los ordenadores personales. Ya en los años 60, se desarrollaron programas educativos e incluso lenguajes de programación como Logo y Pilot. La llegada de los ordenadores personales y la rápida caída de los precios, hizo posible su presencia en centros educativos a finales de los 80. La evolución de los sistemas facilitó su uso y por tanto el acceso a la mayor parte de la población. Pero la inclusión de internet es la pieza definitiva que ha permitido que en muchos hogares haya algún ordenador. El rápido acceso a una cantidad inmensa de información y las amplias posibilidades de intercambio de la misma ha generado en los usuarios la posibilidad de llevar consigo dispositivos para su gestión. 
Otro gran despegue se produjo con el desarrollo de la tableta iPad por Apple. También el software, los programas y datos, están lógicamente sufriendo una importante transformación debido a que los dispositivos móviles actuales poseen microprocesadores y capacidades inferiores a los ordenadores personales. Esto ha dado origen a las actuales APPs, es decir, programas informáticos diseñados para tareas muy específicas con el fin de reducir el volumen de memoria ocupada.

Desde su primer lanzamiento en 2010, iPads y sus APPs se han popularizado en todo el mundo con una amplia gama de usuarios, incluyendo niños preescolares (Costello, 2012). Sin embargo, hay poca investigación empírica que aborda el valor educativo y el impacto de las aplicaciones de iPad que se anuncian como "educativas". Falloon (2013) en un estudio reciente ha investigado las características de diseño y contenido de aplicaciones de iPad seleccionadas en relación con las vías de aprendizaje de niños de cinco años y concluyó que los investigadores y desarrolladores necesitan trabajar juntos para mejorar el valor educativo de las aplicaciones de iPad para niños. De acuerdo con esa premisa nuestro grupo de investigación ha validado software de evaluación e intervención, a partir de proyectos de investigación previos. En efecto, la evaluación computarizada del sentido numérico es ya posible a partir de la versión española del Early Numeracy Test-R estandarizado en nuestro país (Araujo, Aragón, Aguilar, Navarro, \& Ruiz, 2014). Asimismo, se han logrado mejoras en el rendimiento matemático a corta edad a partir de entrenar al alumnado en riesgo con el software "Jugando con Números I y II" (Aragón \& Ruiz, 2015; Navarro, Aguilar, Marchena, Alcalde, \& García, 2010). Determinar el valor educativo de las APPs para estos nuevos dispositivos se presenta como una premisa importante debido a la disponibilidad y popularidad de los dispositivo móviles y Ipads entre los niños pequeños, su uso en muchas escuelas preescolares occidentales (Larkin et al., 2013) y la demanda constante para que los educadores integren mejor la tecnología en el currículo y de este modo fomentar el compromiso activo y el pensamiento de los niños pequeños (Moeller, Fischer, Nuerk, \& Cress, 2015).

\section{OBJETIVOS DE LA INVESTIGACIÓN}

El punto de partida del presente trabajo fue crear y desarrollar un juego divertido para niños de 4 a 7 años con diferentes niveles de dificultad, que tuviese como base el empleo de las nuevas tecnologías. La finalidad pedagógica de la APP que se presenta, consiste en fomentar el desarrollo del conocimiento del número y su relación con la noción de cantidad, así como la estimación posicional del mismo en una recta numérica, considerándose una actividad fundamental para el alumnado en las primeras etapas educativas.

\section{RESULTADOS. LA APP ENCUENTRA EL NÚMERO ESCONDIDO CON MON EL DRAGÓN}

El proceso de creación de la APP Encuentra el número escondido con Mon el Dragón trajo consigo como resultado un instrumento que contribuye a la enseñanza-aprendizaje del conocimiento de los números y de su posición en una línea recta. La tarea de estimación está considerada como un precursor cognitivo de dominio especifico relevante para la mejorar de habilidades matemáticas como el conteo, las operaciones aritméticas o la comprensión de conceptos matemáticos. Asimismo, el dominio de la línea numérica permite al niño responder a preguntas relativas a la magnitud sin hacer referencia a objetos concretos, apoya la regla de modificación de la cardinalidad de un conjunto dependiendo del aumento o sustracción del mismo, y favorece el conocimiento de la posición relativa de un número cuando ésta no puede ser determinada directamente (Gervasoni, 2005; Siegler \& Booth, 2005). En la APP coexisten dos modalidades de estimación, en un caso, aparece un número y el niño debe colocarlo en la posición adecuada en una línea recta (numero-posición) (ver figura 2), o bien, la línea recta tiene una marca distintiva en una en un lugar concreto, sien- 
do el niño el que debe determinar a qué número pertenece ese lugar (posición-número) (ver figura 3). Para esta APP se ha creado un video tutorial debido a la dificultad para comprender la tarea, al que se puede acceder desde la pantalla inicial (ver figura 1).

\section{Figura 1. Pantalla inicial}

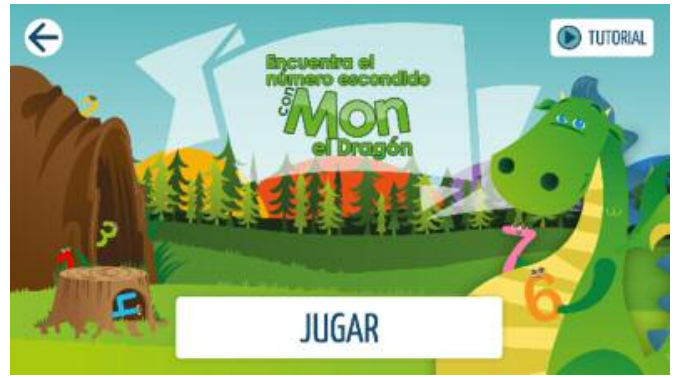

Cada nivel presenta 10 actividades o ítems, debiendo el niño superar un 80\% para pasar al siguiente nivel. En este caso, la corrección de la actividad es a través de un margen de error. Esta se consigue calculando el número que se le pide, menos el número que el niño indica en la recta numérica, dividido por el tamaño de la recta, finalmente el resultado se divide entre 100. Se considera el margen de acierto como el 10\% de desviación de la respuesta correcta, por encima se entiende como error.

Figura 2. Ítem de estimación tipo número-posición

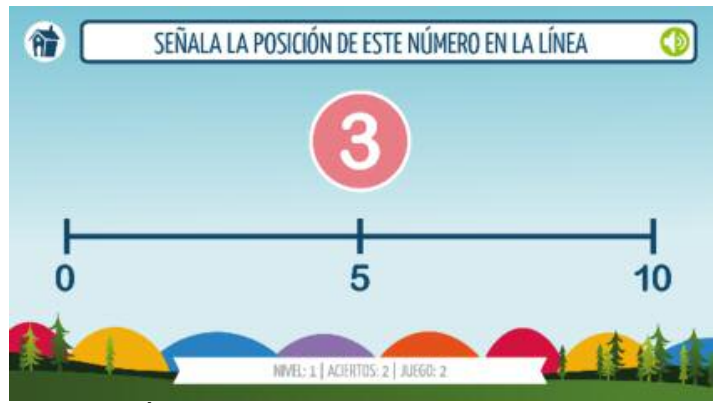

Figura 3. Ítem de estimación posición-número

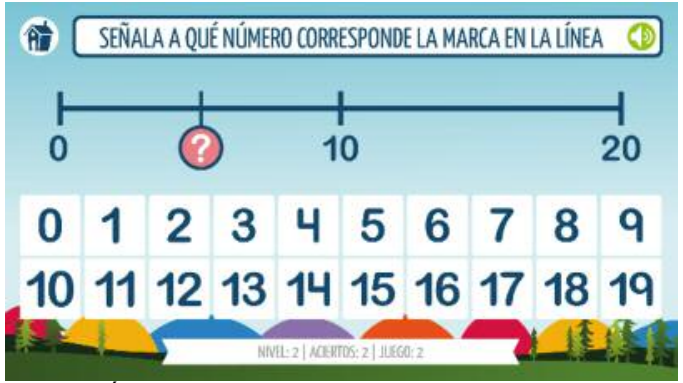




\section{Niveles de dificultad}

Nivel 1. Aparece en la pantalla una línea recta horizontal. En el extremo izquierdo se acompaña de la etiqueta " 0 " y en el extremo opuesto del "10 o 20". De los 10 ítems 0 actividades, las 5 primeras son líneas de 0 a 10 y en las siguientes de 0 a 20. Además, aparece el número central de la línea numérica como ayuda (5 o 10). En un círculo central surge el número objetivo (ver figura 3 ). La actividad consiste en identificar y señalar el lugar que ocupa en la recta el número que se pide en cada actividad.

Nivel 2. Consta de 10 ítems divididos en dos grados de dificultad, como el nivel anterior. Los primeros se muestran en una línea de 0 a 10 y los siguientes de 0 a 20. En ambos casos aparece el número central de referencia y ayuda. También abajo aparecen todos los números desde el 0 al 10 0 20 según la dificultad. En este caso cambia la actividad, ya que la recta aparece con una marca en un lugar concreto acompañada de una incógnita (ver figura 4). El jugador debe indicar a que número corresponde ese lugar, señalando una de las opciones que aparecen abajo.

Nivel 3. En este caso se mezclan las dos modalidades de juego y además se elimina la ayuda de la marca y el número central, aumentando de esa manera la dificultad del juego.

\section{DISCUSIÓN Y CONCLUSIONES}

El objetivo del presente trabajo, fue desarrollar una aplicación para mejorar las habilidades matemáticas tempranas para niños de 4 a 7 años, diseñado para ser utilizado en dispositivos Smartphone y tabletas de tipo iPad. La aplicación fue diseñada para mejorar el conocimiento de la línea numérica y la habilidad de estimación. Dicha destreza está considerada como uno de los predictores específicos más relevantes de la competencia matemática (Colomé et al., 2019; Zhu et al., 2017)

Se espera que un desarrollo de este tipo de aplicaciones educativas, además de mejorar el rendimiento matemático de los niños en edades tempranas, proporcione otros beneficios como la motivación para el aprendizaje matemático debido al empleo de dispositivos tecnológicos de uso diario, como mediadores del proceso de enseñanza-aprendizaje. Actualmente, esta aplicación es una de las 9 aplicaciones que están en fase de pruebas y que, a su vez, son parte de un proyecto de tesis que tratará de estudiar la eficacia del aprendizaje de las matemáticas a una edad temprana a través de las nuevas tecnologías.

$\left(^{*}\right)$ Este trabajo ha sido parcialmente financiado por el proyecto PSI2015-63856-P (MINECO/FEDER)

\section{REFERENCIAS BIBLIOGRÁFICAS}

Aragón, E. L. (2014). Estudio de la eficacia del" e-aprendizaje" a través de la implementación de un programa de entrenamiento en matemática temprana (Doctoral dissertation, Universidad de Cádiz).

Aragón, E. L., Navarro, J. I., Aguilar, M., \& Cerda, G. (2015). Predictores cognitivos del conocimiento numérico temprano en alumnado de 5 años. Revista de Psicodidáctica, 20 (1). doi: 10.1387/RevPsicodidact. 11088

Araújo, A., Aragón, E., Aguilar, M., Navarro, J. I., \& Ruiz, G. (2014). Un estudio exploratorio para la adaptación de la versión española revisada del" Early Numeracy Test-R. European Journal of Education and Psychology, 7 (2). doi: 10.1989/ejep.v7i2.181

Baddeley, A. D., \& Hitch, G. (1974). Working memory. Psychology of Learning and Motivation, 8, 47-89. doi: https://doi.org/10.1016/S0079-7421(08)60452-1

Baddeley, A. D., \& Hitch, G. J. (2000). Development of working memory: Should the Pascual-Leone 
and the Baddeley and Hitch models be merged?. Journal of Experimental Child Psychology, 77(2), 128-137. https://doi.org/10.1006/jecp.2000.2592

Aragón, Navarro, J. I., Aguilar, M., \& Cerda, G. (2014). Cognitive Predictors of 5-Year-Old Students' Early Number Sense // Predictores cognitivos del conocimiento numérico temprano en alumnado de 5 años. Revista de Psicodidactica / Journal of Psychodidactics, 20(1), 83-97. https://doi.org/10.1387/RevPsicodidact.11088

Aragón, E. \& Ruiz, G. (2015). El software «Jugando con números 2.0» y la adquisición del sentido numérico «Playing with numbers 2.0» software and acquisition of number sense. Revista de Psicología y Educación, 10(2), 95-112.

Araujo, A., Aragon, E., Aguilar, M., Navarro, J. I., \& Ruiz, G. (2014). An exploratory study for the standardization of the Spanish version of "Early Numeracy Test-R\{"\} to mathematical learning assessment. European Journal of Education and Psychology, 7(2), 83-93. https://doi.org/10.1989/ejep.v7i2.181

Baddeley, A. D. (2000). The episodic buffer: a new component of working memory? Trends in Cognitive Sciences, 4(11), 417-423. https://doi.org/10.1016/j.apm.2016.02.027

Baddeley, A. D., \& Hitch, G. (1974). Working Memory. In Psychology of learning and motivation, 8, 47-89.

Bartelet, D., Vaessen, A., Blomert, L., \& Ansari, D. (2014). Journal of Experimental Child What basic number processing measures in kindergarten explain unique variability in first-grade arithmetic proficiency? Journal of Experimental Child Psychology, 117, 12-28. https://doi.org/10.1016/j.jecp.2013.08.010

Cirino, P. T. (2011). The interrelationships of mathematical precursors in kindergarten. Journal of Experimental Child Psychology, 108(4), 713-733. https://doi.org/10.1016/j.jecp.2010.11.004

Clements, D., \& Sarama, J. (2009). Learning Trajectories in Early Mathematics - Sequences of Acquisition and Teaching. Encyclopedia of language and Literacy Development, (December), 17.

Costello, S. (2012). My iPad for kids. Indianapolis, indiana. USA: Que.

Cowan, R., \& Powell, D. (2014). The contributions of domain-general and numerical factors to thirdgrade arithmetic skills and mathematical learning disability. Journal of Educational Psychology, 106(1), 214-229. https://doi.org/10.1037/a0034097

De Smedt, B., \& Gilmore, C. K. (2011). Defective number module or impaired access? Numerical magnitude processing in first graders with mathematical difficulties. Journal of Experimental Child Psychology, 108(2), 278-292. https://doi.org/10.1016/j.jecp.2010.09.003

Denton, K., West, J., \& Walston, J. (2003). Reading-Young children's achievement and classroom experiences: Findings from the condition of education . National Center for Education Statistics.

Desoete, A., Roeyers, H., \& De Clercq, A. (2004). Children with Mathematics Learning Disabilities in Belgium. Journal of Learning Disabilities, 37(1), 50-61. https://doi.org/10.1177/00222194040370010601

Falloon, G. (2013). Young students using iPads: APP design and content influences on their learning pathways. Computers and Education, 68, 505-521. https://doi.org/10.1016/j.compedu.2013.06.006

Geary, D. C. (2004). Mathematics and Learning Disabilities. Journal of Learning Disabilities, 37(1), 4-15. https://doi.org/10.1177/00222194040370010201

Geary, D. C., Hamson, C. 0., \& Hoard, M. K. (2000). Numerical and Arithmetical Cognition: A Longitudinal Study of Process and Concept Deficits in Children with Learning Disability. Journal 
of Experimental Child Psychology, 77(3), 236-263. https://doi.org/10.1006/jecp.2000.2561

Gullick, M. M., Sprute, L. A., \& Temple, E. (2011). Individual differences in working memory, nonverbal IQ, and mathematics achievement and brain mechanisms associated with symbolic and nonsymbolic number processing. Learning and Individual Differences, 21(6), 644-654. https://doi.org/10.1016/j.lindif.2010.10.003

Hannula, M. M., Lepola, J., \& Lehtinen, E. (2010). Spontaneous focusing on numerosity as a domain-specific predictor of arithmetical skills. Journal of Experimental Child Psychology, 107(4), 394-406. https://doi.org/10.1016/j.jecp.2010.06.004

Joensen, J., \& Nielsen, H. (2010). More Successful because of Math: Combining a Natural Experiment and a Structural Dynamic Model to Explore the Underlying Channels. Unpublished Working Paper. Recuperado https://www.economicdynamics.org/meetpapers/2011/paper_995.pdf

Kolkman, M. E., Kroesbergen, E. H., \& Leseman, P. P. M. (2013). Early numerical development and the role of non-symbolic and symbolic skills. Learning and Instruction, 25, 95-103. https://doi.org/10.1016/j.learninstruc.2012.12.001

Krajewski, K., \& Schneider, W. (2009). Exploring the impact of phonological awareness, visual-spatial working memory, and preschool quantity-number competencies on mathematics achievement in elementary school: Findings from a 3-year longitudinal study. Journal of Experimental Child Psychology, 103(4), 516-531. https://doi.org/10.1016/j.jecp.2009.03.009

Kroesbergen, E. H., Van Luit, J. E. H., Van Lieshout, E. C. D. M., Van Loosbroek, E., \& Van De Rijt, B. A. M. (2009). Individual differences in early numeracy: The role of executive functions and subitizing. Journal of Psychoeducational Assessment, 27(3), 226-236. https://doi.org/10.1177/0734282908330586

Kroesbergen, E. H., van de Rijt, B., \& van Luit, J. E. H. (2007). Working Memory and Early Mathematics: Possibilities for Early Identification of Mathematics Learning Disabilities. En International Perspectives (Vol. Vol. 30 No, pp. 1-19). Elsevier. https://doi.org/10.1016/S0735004X(07)20001-1

Larkin, K., Purpura, D. J., Ganley, C. M., Larkin, K., Sokolowski, H. M., Ansari, D., ... Rubinsten, 0. (2013). Mathematics Education: Is There an APP For That? Mathematics Education: Yesterday, today and tomorrow (Proceedings of the 36th annual conference of the Mathematics Education Research Group of Australia), 0(1), 426-433. https://doi.org/10.1016/j.jecp.2013.12.009

Laski, E. V, \& Siegler, R. S. (2007). Is 27 a big number? Correlational and causal connections among numerical categorization number line estimation and numerical magnitude comparison. Child Development, 78(6), 1723-1743.

Moeller, K., Fischer, U., Nuerk, H. C., \& Cress, U. (2015). Computers in mathematics education Training the mental number line. Computers in Human Behavior, 48, 597-607. https://doi.org/10.1016/j.chb.2015.01.048

Muñoz, R., Okan, Y., \& Garcia-Retamero, R. (2015). Habilidades numéricas y salud: Una revisión crítica. Revista Latinoamericana de Psicologia, 47(2), 111-123. https://doi.org/10.1016/j.rlp.2015.05.002

Mussolin, C., Mejias, S., \& Noël, M. P. (2010). Symbolic and nonsymbolic number comparison in children with and without dyscalculia. Cognition, 115(1), 10-25. https://doi.org/10.1016/j.cognition.2009.10.006

Navarro, J.I., Aguilar, M., Marchena, E., Alcalde, C., \& García, J. (2010). Assessing early mathematic preschool children. Revista de Educacion, 352(1), 601-615. Recuperado de https://www.scop u s. c $0 \mathrm{~m} / \mathrm{i} n \mathrm{w}$ a $r \mathrm{~d} / \mathrm{r}$ e c o r d. u r i ? e i $d=2-\mathrm{s} 2$. 0 - 
77957745702\& partnerlD=40\&md5=2892342efc1e31d94e74965520778bc6

Navarro, J. I., Aguilar, M., Marchena, E., Ruiz, G., \& Ramiro, P. (2011). Operational development and arithmetic knowledge: Piaget's theory revisited. Revista de Psicodidactica, 16(2), 251-266. Recuperado de https://www.scopus.com/inward/record.uri?eid=2-s2.084856491745\&partnerID=40\&md5=289a16be6dfc9e861814837f2143cc35

Nayfeld, I., Fuccillo, J., \& Greenfield, D. B. (2013). Executive functions in early learning: Extending the relationship between executive functions and school readiness to science. Learning and Individual Differences, 26, 81-88. https://doi.org/10.1016/j.lindif.2013.04.011

Nguyen, T., Watts, T. W., Duncan, G. J., Clements, D. H., Sarama, J. S., Wolfe, C., \& Spitler, M. E. (2016). Which preschool mathematics competencies are most predictive of fifth grade achievement? Early Childhood Research Quarterly, 36, 550-560. https://doi.org/10.1016/j.ecresq.2016.02.003

Núñez-Peña, M. I., Colomé, À., \& Aguilar-Lleyda, D. (2019). Number line estimation in highly mathanxious individuals. British Journal of Psychology, 110(1), 40-59. https://doi.org/10.1111/bjop.12335

OECD. (2016). PISA 2015 Assessment and Analytical Framework. OECD Publishing. https://doi.org/10.1787/9789264255425-en

Purpura, D. J., \& Ganley, C. M. (2014). Working memory and language: Skill-specific or domaingeneral relations to mathematics? Journal of Experimental Child Psychology, 122(1), 104-121. https://doi.org/10.1016/j.jecp.2013.12.009

Randstad. (2016, junio 16). Ciencias, tecnología, ingenierías y matemáticas, las áreas con más oportunidades laborales, pp. 1-4. Recuperado de https://www-randstad-es.s3.amazonaws.com/wpcontent/uploads/2016/07/ciencias-tecnologia-ingenierias-y-matematicas-las-areas-con-masoportunidades-laborales.pdf

Rousselle, L., \& Noël, M. P. (2007). Basic numerical skills in children with mathematics learning disabilities: A comparison of symbolic vs non-symbolic number magnitude processing. Cognition, 102(3), 361-395. https://doi.org/10.1016/j.cognition.2006.01.005

Sarama, J., \& Clements, D. H. (2009). «Concrete» computer manipulatives in mathematics education. Child Development Perspectives, 3(3), 145-150. https://doi.org/10.1111/j.17508606.2009.00095.x

Sasanguie, D., Van den Bussche, E., \& Reynvoet, B. (2012). Predictors for Mathematics Achievement? Evidence From a Longitudinal Study. Mind, Brain, and Education, 6(3), 119-128. https://doi.org/10.1111/j.1751-228X.2012.01147.x

Shalev, R., Manor, O., \& Gross-Tsur, V. (2005). Developmental dyscalculia: a prospective six-year follow-up. Developmental Medicine \& Child Neurology, 47(2), 121-125. https://doi.org/10.1017/S0012162205000216

Siegler, R. S., \& Booth, J. L. (2004). Development of numerical estimation in young children. Child Development, 75(2), 428-444. https://doi.org/10.1111/j.1467-8624.2004.00684.x

Solsona, J., Navarro, J., \& Aguilar, M. (2009). La atención mental en el aprendizaje de la lengua escrita. International Journal of Psycology and Psycological Therapy, 9(3), 335-349.

van Viersen, S., Slot, E. M., Kroesbergen, E. H., van't Noordende, J. E., \& Leseman, P. P. M. (2013). The added value of eye-tracking in diagnosing dyscalculia: A case study. Frontiers in Psychology, 4(OCT), 1-13. https://doi.org/10.3389/fpsyg.2013.00679

Von Aster, M. (2000). Developmental cognitive neuropsychology of number processing and calculation: Varieties of developmental dyscalculia. European Child and Adolescent Psychiatry, 9(SUPPL. 2). https://doi.org/10.1007/s007870070008 
Vujic, A. (2017). Switching on or switching off? Everyday computer use as a predictor of sustained attention and cognitive reflection. Computers in Human Behavior, 72, 152-162. https://doi.org/10.1016/j.chb.2017.02.040

Zhu, M., Cai, D., \& Leung, A. W. S. (2017). Number line estimation predicts mathematical skills: Difference in grades 2 and 4. Frontiers in Psychology, 8(SEP), 1-8. https://doi.org/10.3389/fpsyg.2017.01576 